\title{
Correction to: Leaf stoichiometry is synergistically-driven by climate, site, soil characteristics and phylogeny in karst areas, Southwest China
}

\author{
Yanqiong Li $\cdot$ Wen He $\cdot$ Jin Wu Ping Zhao $(\mathbb{D} \cdot$ Ting Chen $\cdot$ Liwei Zhu \\ Lei Ouyang • Guangyan Ni • Dirk Hölscher
}

Published online: 27 July 2021

(C) Springer Nature Switzerland AG 2021

Correction to: Biogeochemistry

https://doi.org/10.1007/s10533-021-00826-3

In the original version of this article the figure legends of Figs. 3-7 were incorrect. The correct legends are given below. The original article has been updated.

Fig. 3 Leaf traits for different functional groups. Different letters indicate significant differences among different groups for each trait.

The original article can be found online at https:// doi.org/10.1007/s10533-021-00826-3.

Y. Li · P. Zhao $(\bowtie) \cdot$ L. Zhu · L. Ouyang · G. Ni

Key Laboratory of Vegetation Restoration and Management of Degraded Ecosystems, South China Botanical Garden, Chinese Academy of Sciences,

Guangzhou 510650, China

e-mail: zhaoping@scbg.ac.cn

\section{Y. Li}

University of Chinese Academy of Sciences,

Beijing 101408, China

\section{W. He $\cdot$ T. Chen}

Guangxi Key Laboratory of Plant Conservation and Restoration Ecology in Karst Terrain, Guangxi Institute of Botany, Guangxi Zhuang Autonomous Region and Chinese Academy of Sciences, Guilin 541006, China
Fig. 4 Relationships between leaf and soil nutrient concentrations for $\mathrm{N}, \mathrm{P}, \mathrm{K}$, and $\mathrm{Ca}$.

Fig. 5 Relationships between leaf elements and climatic factors in karst areas, Southwest China. Dotted lines were shown if the linear regressions were significant at 0.05 level; $\mathrm{n}$ is the number of samples.

Fig. 6 Individual and shared effects of soil properties (SWC, pH, SOC, soil TN, soil TP, soil TK, soil TCa), site characteristics (longitude, latitude, altitude), climatic factor (MAT, MAP) and leaf features (DM, SLA) on leaf nutrients (N, P, K, Ca) as derived from variation partitioning analysis. (Eigenvalues,

J. $\mathrm{Wu}$

School of Biological Sciences, University of Hong Kong, Hong Kong, China

P. Zhao

Guangdong Provincial Key Laboratory of Applied Botany, South China Botanical Garden, Chinese Academy of Sciences, Guangzhou 510650, China

D. Hölscher

Tropical Silviculture and Forest Ecology, University of Göttingen, 37077 Göttingen, Germany 
contribution rate and the matrix of soil properties and site characteristics were shown in Table S5 and S6).

Fig. 7 SEM for the relationships among climatic factors, plant family composition, and leaf nutrient stoichiometry. The solid and dashed lines represent the positive and negative effects, respectively. The arrow width is proportional to the strength of the relationship. Numbers above the arrows indicate the path coefficients (the pathway was significant at 0.05 level and all the path coefficients were standardized), $\mathrm{R}^{2}$ values are the proportion of variance explained for each variable.

Publisher's Note Springer Nature remains neutral with regard to jurisdictional claims in published maps and institutional affiliations. 